\title{
Mesotelioma pleural em um cão da raça rottweiler
}

\author{
Pleural mesothelioma in a rottweiler dog breed

\begin{abstract}
Andréa Faraon, Ana Claudia Tourrucôo, Kelly Cristini Rocha da Silva Ferreira, Luciana Oliveira de Oliveira \& Rosemari Teresinha Oliveira
\end{abstract}

\begin{abstract}
RESUMO
O mesotelioma é uma neoplasia difusa das membranas serosas da pleura, peritônio, pericárdio e túnica vaginal. É rara em todas as espécies animais. Os sinais clínicos apresentados são secundários ao acúmulo de efusão intracavitária característico deste tipo de tumor. Observam-se dispneia, aumento de volume abdominal, sinais de insuficiência cardíaca congestiva direita, entre outros. O diagnóstico definitivo é obtido através do exame histopatológico. Não existe tratamento efetivo. A cisplatina é a droga de escolha para a terapia paliativa. Neste trabalho é relatado um caso de mesotelioma pleural, diagnosticado na necropsia, em um cão macho da raça rottweiler. A doença apresentou-se rapidamente progressiva, havendo um curto intervalo desde o primeiro atendimento até o óbito do paciente. Clinicamente apresentava muita dispneia em função do acúmulo de efusão hemorrágica intrapleural, sendo a terapia adotada baseada nos sinais clínicos e exames complementares. É, sem dúvida, uma neoplasia com prognóstico bastante desfavorável.
\end{abstract}

Descritores: mesotelioma, pleural, cão, efusão.

\begin{abstract}
Mesothelioma is a diffuse neoplasia of the membranes serous pleura, pericardium, peritoneum and tunica vaginalis. It is rare in all animal species. The clinical signs are secondary to the intracavitary increase of effusion, characteristic of this type of tumor. It is also observed dyspnea, increase of abdominal volume, signs of direct congestive cardiac insufficient, among others. The final diagnosis is achieved with histopathological test. There is no effective treatment. Cisplatin is the drug of choice for palliative therapy. This paper reports a case of pleural mesothelioma, diagnosable in necropsy of a Rottweiler male dog. The disease was progressive, with a brief interval since the first treatment, until the death of the patient. Clinically, the dog appeared to suffer great dyspnea due the increase of intrapleural hemorrhagic effusion. The adopted therapy was based in clinical signs and complementary clinical tests. This neoplasia has, indubitably, an unfavorable prognosis.
\end{abstract}

Keywords: mesothelioma, pleural, dog, effusion. 


\section{INTRODUÇÃO}

O mesotelioma é uma neoplasia derivada de células mesodérmicas da membrana serosa da pleura, do peritônio, do pericárdio e da túnica vaginal do testículo. São tumores de rara ocorrência em animais domésticos e na espécie humana [1,5,9-13,15].

Sua etiologia em medicina veterinária ainda não é comprovada. Em humanos estudos apontam sua relação com a inalação crônica de partículas de amianto [2,5,9-11,15]. Especula-se que cães de proprietários que trabalham com o amianto tenham maior predisposição ao desenvolvimento do tumor [6,7].

Em cães não existe nenhuma predisposição racial ou sexual, o que ocorre em pacientes humanos do sexo masculino $[1,5,6,9,10]$.

Macroscopicamente se observam nódulos de tamanhos variados com distribuição difusa. Raramente ocorre metástase, entretanto, dissemina-se e implanta-se por toda a cavidade $[1,4,5,7,9,11,15]$.

Seu prognóstico é geralmente pobre. Clinicamente observa-se acúmulo constante de fluidos intracavitários responsáveis por promover sinais clínicos de dispneia, aumento de volume abdominal, entre outros [5,9,11,13-15]. Em cães a efusão é geralmente hemorrágica, enquanto que em gatos pode ser quilosa $[3,9,10]$.

A análise citológica do fluido é um exame pouco sensível. O diagnóstico definitivo é feito através de exame histopatológico [4,5,9,10,14,15].

O tratamento do mesotelioma é apenas paliativo. A cisplatina intracavitária reduz a produção de fluidos - pleurodese química. A radioterapia não é uma boa opção em função da apresentação difusa. A pericardiectomia é indicada para evitar o tamponamento cardíaco [1,5,8-10,14,15].

Através deste relato busca-se apresentar a inespecificidade dos sinais clínicos, a dificuldade do diagnóstico in vivo da doença e sua má progressão.

\section{RELATO DO CASO}

Foi atendido no Hospital de Clínicas Veterinárias da Universidade Federal do Rio Grande do Sul um cão da raça rottweiler, macho, com 8 anos de idade, apresentando anorexia, apatia, vômito, diarreia e dispneia. Na ausculta torácica os sons estavam muito abafados. Não havia exame radiográfico disponível nesta data. Foi realizada toracocentese e drenado um volume superior a 1 litro de fluido de aspecto aver- melhado (Figura 1), sendo uma amostra enviada para análise laboratorial. Foram prescritas para administração oral furosemida ${ }^{1}$, aminofilina ${ }^{2}$ e enrofloxacina $^{3}$. Na análise hematológica observou-se anemia moderada e leucocitose, estando os outros parâmetros dentro da normalidade. Clinicamente apresentou grande melhora.

Foi hospitalizado uma semana após o primeiro atendimento. $\mathrm{Na}$ ocasião realizou-se radiografia de tórax após toracocentese, entretanto, permanecia com hidrotórax. A análise citológica do fluido revelou tratar-se de uma neoplasia, porém, não foi possível classificá-la. Em função da falta de especificidade, optou-se pela administração de $0,7 \mathrm{mg} / \mathrm{m}^{2}$ de vincristina $^{4}$ por via intravenosa a cada 7 dias associada a $1 \mathrm{mg} / \mathrm{kg}$ de prednisona ${ }^{5}$ por via oral a cada 24 horas. As medicações já prescritas passaram a ser administradas na forma injetável. Diariamente eram realizadas toracocenteses. O paciente não apresentou melhora clínica com o tratamento e foi a óbito em 5 dias.

$\mathrm{Na}$ necropsia foram observados inúmeros nódulos de tamanhos variados recobrindo por completo a pleura (figura 2). O exame histopatológico revelou tratar-se de um tumor mesenquimal maligno, o mesotelioma.

\section{DISCUSSÃO}

O mesotelioma é uma neoplasia que promove grandes acúmulos de fluídos intracavitários de aspecto hemorrágico [5,9,11,13-15]. Repetidas toracocenteses foram realizadas para promover melhora na capacidade respiratória do paciente.

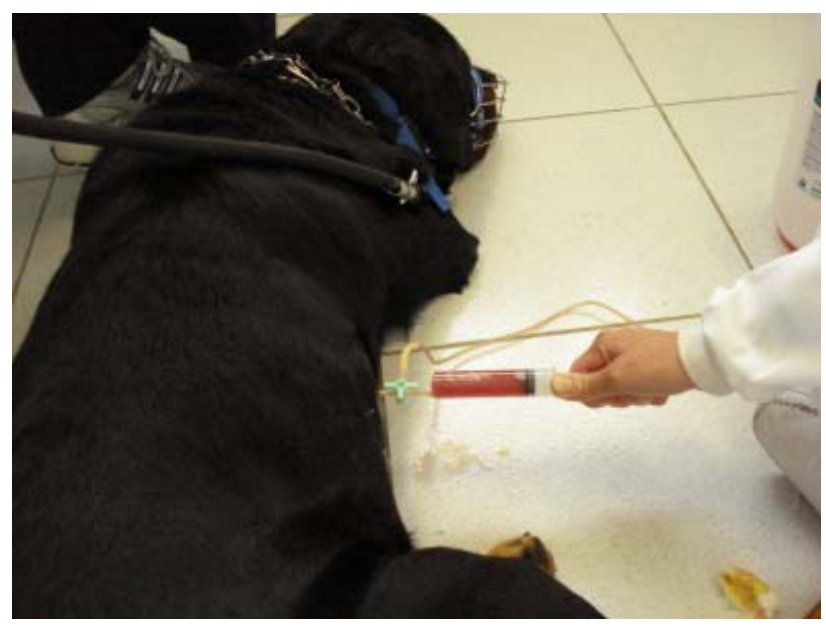

Figura 1. Toracocentese de efusão hemorrágica em um cão com tumoração pleural, apresentando grave dispneia. 
A citologia é um método pouco sensível para o diagnóstico do mesotelioma em função da dificuldade na diferenciação das células tumorais de células mesoteliais reativas. O diagnóstico definitivo é dado através da histopatologia $[4,5,9,10,14,15]$. No caso relatado o diagnóstico foi obtido somente com análise histopatológica de fragmentos coletados na necropsia.

O mesotelioma dissemina-se por toda a cavidade e apresenta-se sob a forma de nódulos de vari-

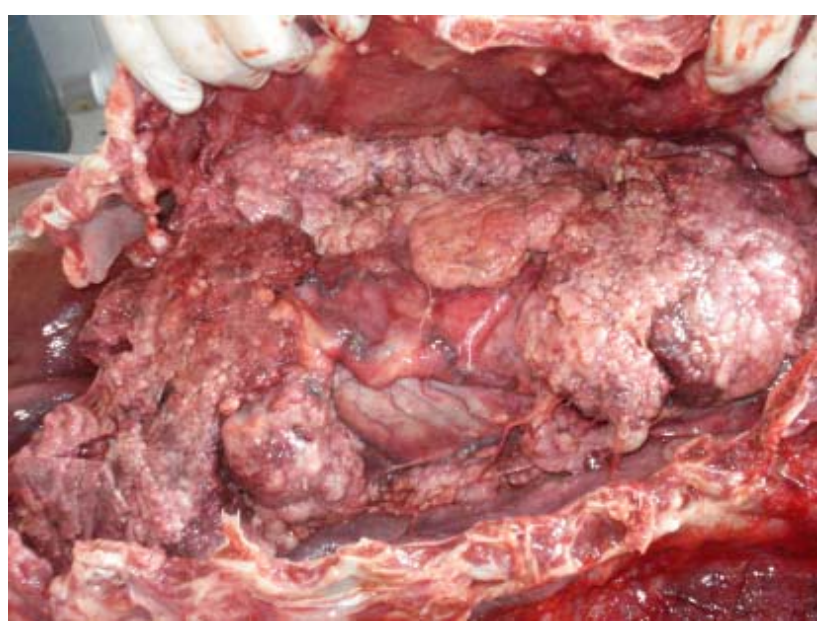

Figura 2. Imagem de necropsia. Visão do interior da cavidade torácica repleta de nódulos de tamanhos diversos (mesotelioma pleural). ados tamanhos $[1,4,5,7,9,11,15]$. Na necropsia as massas tumorais apresentavam-se completamente disseminadas sobre toda a membrana pleural e seu aspecto, como referido pelos autores, era de nódulos com tamanhos diversos.

A cisplatina é a droga de eleição para o tratamento paliativo do mesotelioma. Essa droga, administrada no interior da cavidade comprometida, promove o colabamento das membranas impedindo o acúmulo da efusão produzida a partir do tumor $[5,8,9,12,14,15]$. Não foi instituída terapia com cisplatina devido a rápida evolução do quadro clínico e da ausência de um diagnóstico preciso.

Certamente mais estudos são necessários para que se determine a etiologia e melhores opções terapêuticas, mesmo sendo essa neoplasia de rara ocorrência e de prognóstico desfavorável.

\section{NOTAS INFORMATIVAS}

${ }^{1}$ Lasix, Sanofi-Aventis Farmacêutica Ltda. Suzano, SP, Brasil. ${ }^{2}$ Aminofilina, Sandoz -Novartis Biociências S.A. São Paulo, SP, Brasil

${ }^{3}$ Baytril, Bayer S.A. São Paulo, SP, Brasil.

${ }^{4}$ Vincristina, Pharmacia Ind. Com. Ltda. Botafogo, RJ, Brasil. ${ }^{5}$ Meticortem, Mantecorp Ind. Com. Ltda. Rio de Janeiro, RJ, Brasil.

\section{REFERÊNCIAS}

1 Andrade J.M., Diniz P.P., Silva C.V., Mucha C.J. \& Camacho A.A. 2003. Doenças pericárdicas no cão: revisão. Clínica Veterinária. 42: 42-50.

2 Andrews E. J. 1973. Pleural mesothelioma in a cat. Journal of Comparative Pathology. 83(2): 259-263.

3 Aronsohn M.G. \& Carpenter J.L. 1999. Surgical treatment of idiopathic pericardial effusion in the dog: 25 cases (19781993). Journal of the American Animal Hospital Association. 35(6): 521-525.

4 Belerenian G.C. 2003. Afecções do pericárdio. In: Belerenian G.C., Mucha C.J. \& Camacho A.A. (Eds). Afecções cardiovasculares em pequenos animais. São Caetano do Sul: Interbook Ltda., pp.194-197.

5 Costa F.S., Tostes R. A., Andrade S. F. \& Farias M. R. 2002. Mesotelioma peritoneal em um cão: relato de caso. Clínica Veterinária. 38: 45-49.

6 Enzinger F.M. \& Weiss S.W. 1985. Mesotelioma. In: Enzinger F.M. \& Weiss S.W. (Eds). Tumores de tejidos blandos. Buenos Aires: Médica Panamericana, pp.592-622.

7 Garrett L.D. 2007. Mesothelioma. In: Withrow S.J. \& Vail D.M. (Eds). Small animal clinical oncology. 4th edn. Canada: Saunders, pp.804-808.

8 Lanore D. \& Delprat C. 2004. Quimioterapia Anticancerígena. São Paulo: Roca, 228p.

9 Morris J. \& Dobson J. 2001. Tratament opitions. In: Morris J. \& Dobson J. (Eds) Small animal oncology. London: Blackwell Science, pp.31-49.

10 Morrison W.B. 1998. Nonpulmonary intrathoracic cancer. In: Morrison W.B. (Ed). Cancer in dogs and cats: medical \& surgical management. Baltimore: Willanms \& Wilkins, pp.537-549.

11 Piacenti A.M., Ocarino N.M., Silva A.E., Rachid M.A., França S.A. \& Serakides R. 2004. Mesotelioma pleural com metástase renal em gato. Arquivo Brasileiro Medicina Veterinária e Zootecnia. 56(4): 558-561. 
12 Serakides R., Cassali G.D., Sant’Ana F.F. \& Nascimento E.F. 2001. Mesotelioma peritoneal em cão: relato de caso. Arquivo Brasileiro Medicina Veterinária e Zootecnia. 53(2): 183-187.

13 Terra R.M., Teixeira L.R., Beyruti R., Takagaki T.Y., Vargas F.S. \& Jatene F.B. 2008. Mesotelioma pleural maligno: experiência multidisciplinar em hospital público terciário. Jornal Brasileiro de Pneumologia. 34(1): 13-20.

14 Tilley L.P. \& Smith Jr. F.K. 2003. Consulta Veterinária em 5 Minutos: Espécies Canina e Felina. 2.ed. São Paulo: Manole, pp.956-957.

15 White R.N., Ducan B. \& Lascelles X. 2004. Tumours of the respiratory sytem and thoracic cavity. In: White R.N., Ducan B. \& Lascelles X. (Eds). BSAVA manual of canine and feline oncology. Australia: Blackwell, pp.269-271. 